\title{
Gas Holding mechanism and Impeller Structure Optimization of High Efficiency Gas-Liquid Mixer
}

\author{
Jing Rui $\mathrm{Li}^{1}$, Ting Jun Yan ${ }^{1, \mathrm{a}}$ and Bai Ru Zhang ${ }^{1}$ \\ ${ }^{1}$ Mechanical and Electrical Engineering Institute, Beijing University of Chemical Technology, Beijing 100029, China
}

\begin{abstract}
This research aims at researching one high efficiency gas-liquid mixer which can be applied under high gas content condition and can overcome the limited application of sedimentation and rotary oil-gas separator for electric submersible pump. Standard k- $\varepsilon$ model and population balance model of the Fluent are applied to simulate the gas-liquid two-phase flow in high efficiency gas-liquid mixer. The internal flow field of impeller opening with different radius and amounts are simulated. The gas holding mechanism of high efficiency gas-liquid mixer is also analyzed. Then according to the gas particle size at the output of impeller, the optimized structure parameter can be obtained. The simulation results show that part of the high pressure liquid at impeller outlet would form cross flow with fluid in normal flowing inside the impeller passage to produce strong shear friction inside the area and burst a large amount of bubbles accumulated in the area. Also, it is proved to be more beneficial for breaking bubble and dealing with gas under the circumstances that gas content at the input is higher than $35 \%$, opening diameter of impeller is $\Phi 4.8 \mathrm{~mm}$ and opening amount of impeller is 2 .
\end{abstract}

\section{Introduction}

Generally, the output liquid of the oilfield contains natural gas, water and the other substance. If the content of the gas in the well fluid exceeds designed allowable value of the ESP, it can result in the significant reduction of displacement, head and efficiency of electric submersible pump, so that the working performance can be impacted [1-3]. Aiming at improving applicability of the ESP in live wellbore, it is general to add a gas-processing equipment (oil-gas separator) at its input. However, the normal ESP can not work effectively if gas content is higher than 35\% [4-5]. In this case, one high efficiency gas-liquid mixer (e.g. from Schlumberger) can solve the problem and improve the performance of the ESP [6]. In China, several ESP production companies such as Chittagong Machinery Manufacturing Company, Victory Pump Company, Daqing oilfield power of God have produced gas-processing equipment, which is effectively applicable for high gas content considering the related productions in other countries. Henceforth, researching this lack plays an important role for extracting oil in high gas content oil well.

\footnotetext{
a Corresponding author: Ting Jun Yan: yantj555@163.com.
} 


\section{Gas holding mechanism of high efficiency gas-liquid mixer}

\subsection{The impeller structure of high efficiency gas-liquid mixer}

High efficiency gas-liquid mixer is installed in the entrance of the ESP and its structure is similar to multistage centrifugal pump, which is composed of multistage impeller. The impeller structure is showed in figure 1. The impeller has 6 blades, and the low pressure area of the impeller near the root of each blade is provided with two equal diameter holes. In figure 1, the holes are labeled as A and B, and the two holes are arranged along the flow direction of the blade, the total amount of holes is 12 .

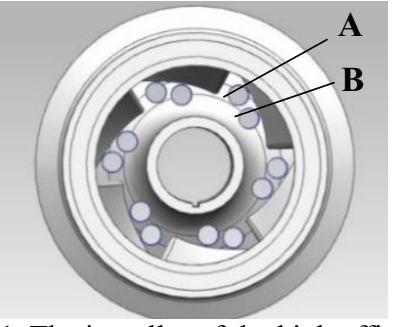

Figure 1. The impeller of the high efficiency gas-liquid mixer

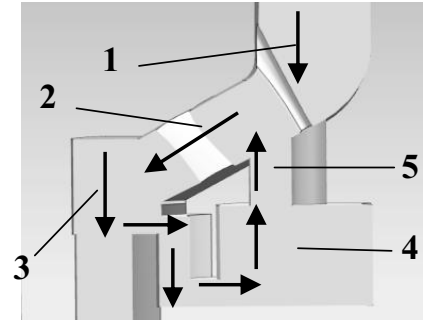

Figure 2. The flow channel section of the high efficiency gas-liquid mixer cross hole A

Figure 2 shows the flow passage section of the high efficiency gas-liquid mixer cross hole A. The flow passage section is composed of the flow passage 1 of impeller entrance, the rotating impeller passage 2, impeller outlet 3, the reflux cavity 4 and the flow passage 5 of holes A. The hole A is connected with the reflux cavity and the impeller entrance. The direction of the arrow in the figure shows the direction of the fluid which flows in the impeller of the high efficiency gas-liquid mixer. The fluid enters into the impeller passage 2 through the impeller entrance 1 , then outflows from the outlet 3 of the impeller, into the roulette. Among them, there is a small amount of fluid flow along the clearance from the impeller outlet 3 to the back of the impeller, flow through the reflux cavity 4 and roulette hole $\mathrm{A}$ and $\mathrm{B}$, return to the impeller inlet port 1 .

\subsection{Liquid phase velocity and pressure distribution of high efficiency gas-liquid mixer}

According to the impeller structure of high efficiency gas-liquid mixer, standard k- $\varepsilon$ model and population balance model in Fluent software can be used to simulate the internal flow field of the impeller. Figure 3 shows the liquid phase velocity vector in the high efficiency gas-liquid mixer through hole A and hole $\mathrm{B}$. The discussion of high efficiency gas-liquid mixer mixing mechanism is the preliminary studies of our group which have been published [7]. As shown in Figure 3a, the flow velocity in the reflux cavity and hole $\mathrm{A}$ is large and flowing uniformity. However, the flow velocity in the reflux cavity and hole B is small and flowing weakly as shown in figure $3 \mathrm{~b}$. Thus, due to the hole A approaches the impeller outlet and the hole B is located at the impeller inlet, when the fluid flows through the hole A and hole B, the resistance of flow passage of hole A is relatively small, so that most of the return fluid flows to the impeller inlet through the flow passage of hole A, while a small amount of fluid flows through the flow passage of hole B.

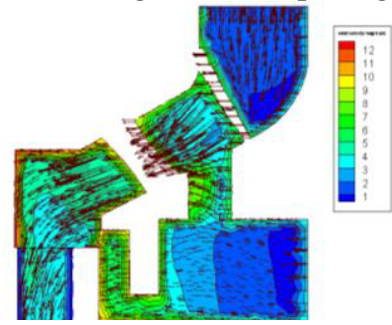

a. The picture of velocity vector through hole A

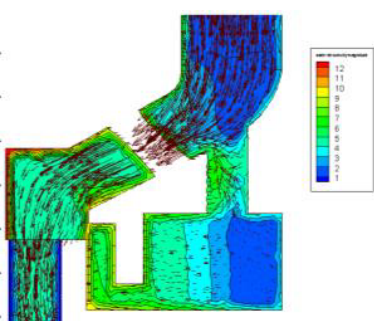

b. The picture of velocity vector through hole B

Figure 3. Liquid phase velocity vector in the high efficiency gas-liquid mixer 


\subsection{Gas-liquid mixing principle of high efficiency gas-liquid mixer}

Figure 4 and Figure 5 show the fluid velocity vector and the turbulent kinetic energy contours of the high efficiency gas-liquid mixer impeller near the openings. A part of the high pressure fluid flows through the high efficiency gas-liquid mixer roulette hole A and B, and then flows to the impeller inlet port back. The arrows direction of I and II regional showed in FIG. 4 is the fluid velocity direction within roulette holes (A, B). The flow direction and movement direction of the normal flow of fluid in the impeller passage formed cross flow because there is a certain Angle. So there is relative strong shearing action between two parts of fluid. When shear force is greater than the surface tension of the air bubble in the fluid, the large diameter of bubble gathered here is torn and broken into smaller bubbles. Figure 5 shows the high velocity of the fluid at the openings so that the turbulent kinetic energy near the openings is large, so small bubbles mixed with the surrounding liquid by strong turbulent action. Through cutting and mixing after flowing out of the roulette holes (A, B), the gas particle size will be decreased and the distribution of gas and liquid is uniform, as similar as single-phase flow, thus can improve the electric submersible pump suction condition, provides guarantee for stable operation of the electric submersible pump.

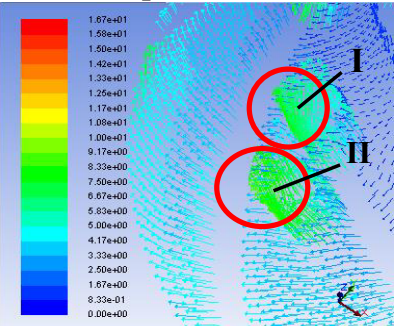

Figure 4. The fluid velocity vector of the high efficiency gas-liquid mixer impeller near the openings

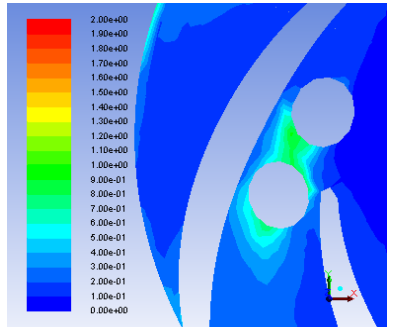

Figure 5. The turbulent kinetic energy contours of the high efficiency gas-liquid mixer impeller near the openings

\section{The mixing effect impacted by the impeller parameters of high efficiency gas-liquid mixer}

Simulate the flow field of gas-liquid two-phase mixing process within the high efficiency gas-liquid mixer by using Fluent. The unstructured mesh arrangement with hexahedral elements is used in calculating watershed. The periodic boundary is applied to the circumferential of impeller. Velocity inlet is set for entry of computation region and free flow condition is inducted for outlet. Standard k- $\varepsilon$ model is used to serve as a turbulent flow model with the object of simulate being the gas-liquid two-phase flow. The population balance model is implemented in the two-fluid model that accounts for the coalescence and breakup of bubble. According to the oil field, choose to use 98 pumps which is used widely, the other calculation parameters are: high efficiency gas-liquid mixer operating pressure is $6 \mathrm{MPa}$ (submergence depth is $600 \mathrm{~m}$ ), the air as the gas phase, the water as the liquid phase, inlet velocity $1.5 \mathrm{~m} / \mathrm{s}$ (Q is $210 \mathrm{~m}^{3} / \mathrm{d}$ ), the impeller speed $2850 \mathrm{r} / \mathrm{min}$, the initial bubble diameter is set to $\Phi 1 \mathrm{~mm}$.

\subsection{The influence of high efficiency gas-liquid mixer impeller opening diameter}

The size of the opening diameter of the impeller would affect the performance of the high efficiency gas-liquid mixer. High efficiency gas-liquid mixers with the opening diameter respectively of $\Phi 2 \mathrm{~mm}$, $\Phi 3 \mathrm{~mm}, \Phi 4 \mathrm{~mm}, \Phi 4.8 \mathrm{~mm}, \Phi 5 \mathrm{~mm}$ and $\Phi 6 \mathrm{~mm}$ were compared. And as the inlet gas rates are respectively $5 \%, 10 \%, 15 \%, 20 \%, 25 \%, 30 \%, 35 \%, 40 \%, 45 \%$, we found that under different inlet gas rates, the internal flow field distribution of high efficiency gas-liquid mixer with 6 different kinds of opening diameter is similar. Here inlet gas rate is selected as $15 \%$ and the gas particle size distribution on the flow channel section of 6 kinds of high efficiency gas-liquid mixers is analyzed. 


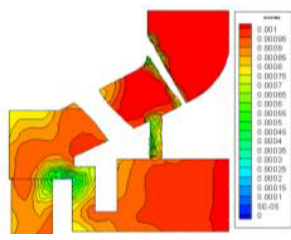

(a) $2 \mathrm{~mm}$

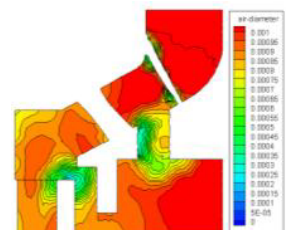

(e) $5 \mathrm{~mm}$

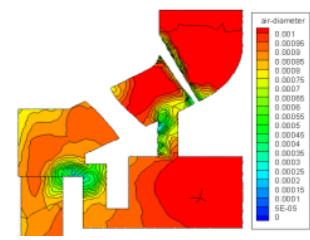

(b) $3 \mathrm{~mm}$

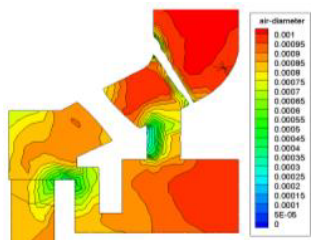

(d) $4.8 \mathrm{~mm}$

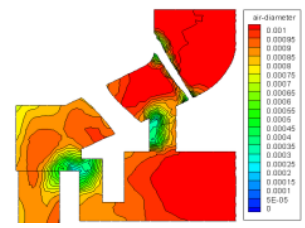

(c) $4 \mathrm{~mm}$

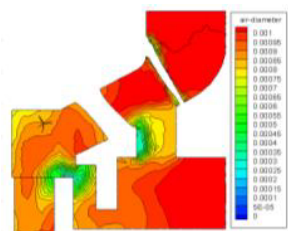

(f) $6 \mathrm{~mm}$

Figure 6. The gas diameter contours of the high efficiency gas-liquid mixer with different aperture openings

Figure 6 shows the gas diameter contours on the cross hole A section of the high efficiency gas-liquid mixer with different aperture openings. Each of the flow channel's cross-sections is corresponding to Figure 2. From the gas diameter contours it can be seen that when the high efficiency gas-liquid mixer aperture opening is respectively $\Phi 2 \mathrm{~mm}, \Phi 3 \mathrm{~mm}, \Phi 4 \mathrm{~mm}, \Phi 4.8 \mathrm{~mm}, \Phi 5 \mathrm{~mm}$ and $\Phi 6$ $\mathrm{mm}$, the average gas particle diameter of the impeller's outlet was respectively: $\Phi 0.806 \mathrm{~mm}, \Phi 0784$ $\mathrm{mm}, \Phi 0780 \mathrm{~mm}, \Phi 0.740 \mathrm{~mm}, \Phi 0.790 \mathrm{~mm}, \Phi 0.786 \mathrm{~mm}$. It's found that the high efficiency gas-liquid mixer with $\Phi 4.8 \mathrm{~mm}$ opening diameter has the least average gas particle diameter of the impeller's outlet compared to the other 5 kinds, which illustrates that the opening diameter of $\Phi 4.8 \mathrm{~mm}$ is more beneficial to bubble's broken.

\subsection{The influence of efficient gas-liquid mixer impeller opening number}

As for the effect of the number of openings on the performance of high efficiency gas-liquid mixer, according to the results of the discussion, through exploring the size of the gas particle of the impeller outlet with single hole and double holes (each opening hole area is same). Therefore, the optimal number of openings can be determined.
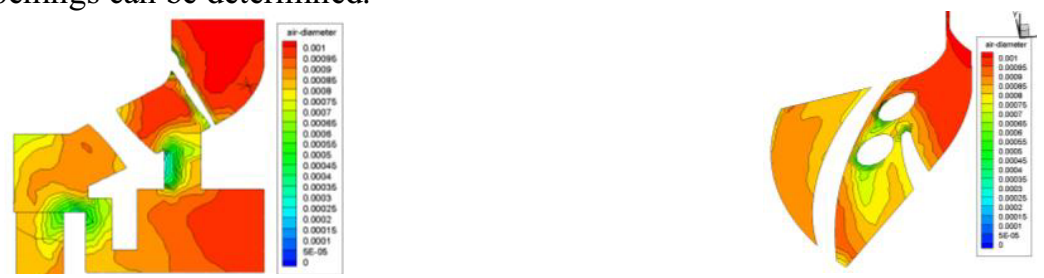

Figure 7. The gas diameter contours of the high efficiency gas-liquid mixer with two holes in axial and radial section of the flow path cross hole A
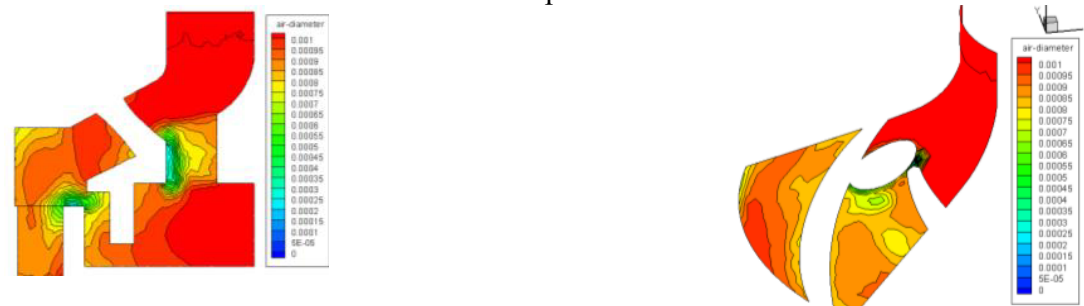

Figure 8. The gas diameter contours of the high efficiency gas-liquid mixer with one holes in axial and radial section of the flow path cross hole A

Figure 7 shows the gas diameter contours of the high efficiency gas-liquid mixer with two holes in axial and radial section of the flow path cross hole A. Figure 8 shows the gas diameter contours of the high efficiency gas-liquid mixer with one hole in axial and radial section of the flow path cross hole A. According to the gas particle size distribution of the high efficiency gas-liquid mixer with double and 
single hole, the average gas particle size of the impeller outlet is $\Phi 0.746 \mathrm{~mm}$ and $\Phi 0.811 \mathrm{~mm}$. By contrasting, it's found that the high efficiency gas-liquid mixer with double holes had smaller gas average particle size at impeller outlet, thus the two-hole high efficiency gas-liquid mixer is more conducive to bubble broken.

\section{Conclusion}

1. The high pressure liquid at impeller outlet would return to the zone on the blade non-working surface with the minimum pressure through the opening on the high efficiency gas-liquid mixer, and form cross flow with fluid in normal flowing inside the impeller passage, thereby dispersing, chopping the bubbles, and mixing the bubbles with the liquid.

2. The opening radius is $4.8 \mathrm{~mm}$, the opening number is 2 , more conducive to the bubble breaking, the gas treatment effects in better.

\section{References}

1. H. Pineda, J. Biazussi, F. Lopez, et al. Phase distribution analysis in an Electrical Submersible Pump (ESP) inlet handling water-air two-phase flow using Computational Fluid Dynamics (CFD)[J]. Journal of Petroleum Science and Engineering. 2016; 139: 49-61.

2. H Stel, T Sirino, F J Ponce, et al. Numerical investigation of the flow in a multistage electric submersible pump[J]. Journal of Petroleum Science and Engineering. 2015; 136: 41-54.

3. H Q Zhao, Z L Li, H Y Sun. Numerical Simulation of Flow Field in Downhole Hydrocyclone Oil-gas Separator[J]. Journal of China University of Petroleum (Edition of Natural Sciences), 2007; 31(4): 94-97.

4. H P Tang, Q B Wang, G Y Yang, et al. A Honeycomb-Structured Ti-6Al-4V Oil-Gas Separation Rotor Additively Manufactured by Selective Electron Beam Melting for Aero-engine Applications[J]. JOM. 2016; 68(3): 799-805.

5. Z L Li, H Y Sun. Performance Simulation Test of Downhole Centrifugal Cyclone and High Efficiency Oil-gas Separator[J]. China Petroleum Machinery. 2007; 35(12): 5-8.

6. Y.L Woon, Bartlesville, OKla. Downhole Pumping System for Recovering Liquids and Gas: United States, 5628616[P]. 1997-05-13.

7. B R Zhang, T J Yan, J Li, et al. Theoretical Research on Gas Holding of a New Kind Mixer with High Gas-liquid Ratio[J]. China Petroleum Machinery, 2016, 44(9): 52-57. 